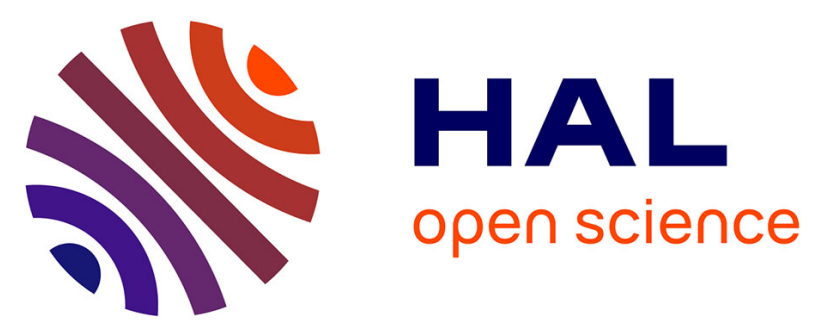

\title{
A Fourier transform spectrometer without a beam splitter for the vacuum ultraviolet range: From the optical design to the first UV spectrum
}

Nelson de Oliveira, Denis Joyeux, Daniel Phalippou, Jean-Claude Rodier, François Polack, Michel Vervloet, Laurent Nahon

\section{To cite this version:}

Nelson de Oliveira, Denis Joyeux, Daniel Phalippou, Jean-Claude Rodier, François Polack, et al.. A Fourier transform spectrometer without a beam splitter for the vacuum ultraviolet range: From the optical design to the first UV spectrum. Review of Scientific Instruments, 2009, 80 (4), pp.043101. 10.1063/1.3111452 . hal-00648580

\section{HAL Id: hal-00648580 \\ https://hal-iogs.archives-ouvertes.fr/hal-00648580}

Submitted on 8 Jul 2013

HAL is a multi-disciplinary open access archive for the deposit and dissemination of scientific research documents, whether they are published or not. The documents may come from teaching and research institutions in France or abroad, or from public or private research centers.
L'archive ouverte pluridisciplinaire HAL, est destinée au dépôt et à la diffusion de documents scientifiques de niveau recherche, publiés ou non, émanant des établissements d'enseignement et de recherche français ou étrangers, des laboratoires publics ou privés. 


\title{
A Fourier transform spectrometer without a beam splitter for the vacuum ultraviolet range: From the optical design to the first UV spectrum
}

\author{
N. de Oliveira, ${ }^{1, a)}$ D. Joyeux, ${ }^{2}$ D. Phalippou, ${ }^{2}$ J. C. Rodier, ${ }^{2}$ F. Polack, ${ }^{1}$ M. Vervloet, ${ }^{1}$ and \\ L. Nahon ${ }^{1}$ \\ ${ }^{1}$ Synchrotron Soleil, Orme des Merisiers, St AUBIN BP 48, 91192 GIF sur Yvette CEDEX, France \\ ${ }^{2}$ Laboratoire Charles Fabry de l'Institut d'Optique, RD 128, Campus Polytechnique, \\ PALAISEAU cedex, France
}

(Received 22 December 2008; accepted 11 March 2009; published online 13 April 2009)

\begin{abstract}
We describe a Fourier transform (FT) spectrometer designed to operate down to $60 \mathrm{~nm}(20 \mathrm{eV})$ on a synchrotron radiation beamline for high resolution absorption spectrometry. As far as we know, such an instrument is not available below $140 \mathrm{~nm}$ mainly because manufacturing accurate and efficient beam splitters remains a major problem at these wavelengths, especially if a wide bandwidth operation is desired. In order to overcome this difficulty, we developed an interferometer based on wave front division instead of amplitude division. It relies on a modified Fresnel bimirror configuration that requires only flat mirrors. The instrument provides path difference scanning through the translation of one reflector. During the scanning, the moving reflector is controlled by an optical system that keeps its direction constant within a tolerable value and provides an accurate interferometric measurement of the path difference variation. Therefore, a regular interferogram sampling is obtained, producing a nominal spectral impulse response and an accurate spectral calibration. The first results presented in this paper show a measured spectral resolution of $\delta \sigma$ $=0.33 \mathrm{~cm}^{-1}$ (interval between spectral samples). This was obtained with a sampling interval of 29 $\mathrm{nm}$ (path difference) and $512 \mathrm{~K}$ samples from a one-sided interferogram using a cosine FT. Such a sampling interval should allow the recording of large bandwidth spectra down to $\lambda=58 \mathrm{~nm}$ with an ultimate resolving power of 500000 at this wavelength. In order to check the instrument performances, we first recorded an interferogram from a He-Ne stabilized laser. This provided the actual spectral impulse function, which was found to be fully satisfactory. The determination of the impulse response distortion and of the noise on the vacuum ultraviolet (VUV) spectral range provided accurate information in the sampling error profile over a typical scan. Finally, the instrument has been moved to the SU5 undulator-based synchrotron radiation beamline (Super-ACO facility, LURE, Orsay, France). A high resolution spectrum of $\mathrm{O}_{2}$ (the Schumann-Runge absorption bands, 185-200 nm) was computed from recorded interferograms using the beamline monochromator at the zeroth order to feed the instrument with an $11 \%$ relative bandwidth "white" beam (2003). These UV measurements are very close to those found in the literature, showing nominal performances of the FT spectrometer that should translate into an unprecedented resolving power at shortest VUV wavelengths. A recent upgrade (2007) and future developments will be discussed in light of the current installation of the upgraded FT spectrometer as a permanent endstation for ultrahigh resolution absorption spectrometry on the VUV beamline DESIRS at SOLEIL, the new French third generation synchrotron facility. (C) 2009 American Institute of Physics. [DOI: 10.1063/1.3111452]
\end{abstract}

\section{INTRODUCTION}

The advantages of Fourier transform spectroscopy (FTS) compared to grating-based spectroscopy are well known. Multiplex and throughput advantages have contributed to FTS success from the infrared to UV spectral range during the last 3 or 4 decades. However, the multiplex (or Felgett) advantage disappears or becomes less significant at shorter wavelengths (when noise becomes photon-limited). As for the throughput (or Jacquinot) advantage, it is related to amplitude division interferometers, which can no longer be used

\footnotetext{
${ }^{a)}$ Author to whom correspondence should be addressed. Electronic mail:
} nelson.de.oliveira@synchrotron-soleil.fr. in the vacuum ultraviolet ${ }^{1}$ (VUV) because of the lack of accurate and efficient beam splitters at short wavelength, especially for wide band operation. Nevertheless, FTS remains attractive in these conditions (i.e., below $140 \mathrm{~nm}$ ) because of its ability to reach very high resolving power and also of its intrinsic accuracy in wavelength and amplitude scaling.

A typical FTS instrument is based on a Michelson interferometer in which one mirror is moved to vary the optical path difference (OPD) between the interfering beams. One records the interferometric signal versus OPD to get the interferogram. The spectrum is then recovered from the interferogram by an inverse FT operation. The resolving power $R=\sigma / \delta \sigma$ is related to the maximum path difference $\left(\Delta_{\max }\right)$ 
reached by the interferometer. A standard definition of the theoretical resolving power is the number of waves of incident light in $2 \Delta_{\max }: R_{S}=2 \Delta_{\max } / \lambda$. One can also address the full width at half maximum (FWHM) width of the impulse response. Assuming a perfect system with no apodization, the impulse response is a sinc function, and the corresponding resolution is found to be 1.2 times smaller, i.e., $R_{\text {FWHM }}$ $=R_{S} / 1.2$. Other criterion may be used depending on context and yields a different value, always in the form of $R_{S} / a$, with an $a$ numerical coefficient greater than and close to one. ${ }^{2}$ For the sake of simplicity we shall use $R_{S}$ unless otherwise mentioned.

The FTS can achieve resolving power greater than 1 $\times 10^{6}$ in the VUV region down to $\lambda=140 \mathrm{~nm}$ (Ref. 3) (about $8.8 \mathrm{eV}$ ). Below this limit (which we could name the "beam splitter limit"), FTS is missing and as a matter of fact the only existing high resolution spectroscopic techniques are based on VUV tunable laser sources or grating spectrometers.

Tunable coherent laser sources, based on various fourwave mixing schemes, can now cover the VUV range of up to about $20 \mathrm{eV}(62 \mathrm{~nm})$ and provide extremely high resolving power of up to $10^{7} .^{4}$ Of course, the laser technique is very much a line by line method and is not suitable for spectra study covering a broad spectral range since its extended operation is very tedious, requiring several changes in the setup (dyes, mirrors).

The other major spectrometry technique in the VUV is based on the use of gratings. With grating spectroscopy, the ultimate resolving power is constant and limited by the number of grooves coherently illuminated and therefore by the optical quality that can be achieved on large gratings. Nowadays, manufacturers cannot guarantee slope errors below about $1 \mu \mathrm{rad}$; this means in practice that grating spectroscopy can hardly reach $2 \times 10^{5}$ resolving power in the VUV. $^{5-7}$ Such ultimate performances have been reached at Super-ACO (LURE, Orsay) with the SU5 undulator-based beamline equipped with a high resolution scanning grating monochromator with which $10^{5}$ was routinely achieved in the VUV range of up to about $21 \mathrm{eV}^{5}$ On the other hand, the FTS resolving power is proportional to the maximum OPD that can be achieved (only related to the scanning range of the interferometer) and $\sigma$. This means that FTS resolving power increases linearly with the energy by opposition with grating-based spectrometers that give a constant resolution $\delta \lambda$ so that the actual resolving power $(\lambda / \delta \lambda)$ decreases with $\lambda$. Therefore at short wavelengths, FTS may provide much higher resolving power than grating-based spectrometers. Another series of advantages of the FTS compared to grating-based spectrometers lies in the way the interferometric signal is recorded, i.e., in a wavelength-multiplex way over a very large spectral range instead of step by step. This makes the acquisition of a spectrum, for a given signal to noise ratio $(\mathrm{S} / \mathrm{N})$ much faster in the case of a large studied spectral range. Besides, a spectrum acquired with a FTS is quite insensitive to experimental conditions fluctuations such as the column density since all lines are altered at the same level. Together with the fact that the whole spectrum is acquired with a constant $\mathrm{S} / \mathrm{N}$, regardless of source intensity fluctuations make FTS an ideal tool to extract precise absorption cross sections. Finally, FTS shows a strict linearity in the wavelength scale; as a result, one can put the spectrum on an absolute scale using a single reference wavelength in the source. In contrast, grating-based spectrometers require a series of reference throughout the spectrum; this could be difficult, especially in the VUV where laser lines measurements are rare or even absent in the far VUV. Note that synchrotron radiation (SR)-based sources using a grating monochromator as well as laser-based techniques can be used for all type of nonphotonic spectroscopies, including, for instance, various electron and ion spectroscopies, while FTS is limited to absorption (or emission) spectroscopy, i.e., direct photon spectroscopy.

Nevertheless, there is a very large demand from the scientific community for broadband, very high resolution absorption spectroscopy in the VUV at wavelength shorter than the beam splitter limit, say, below $140 \mathrm{~nm}$ and for which FTS would be the ideal tool. The FTS Group at Imperial College of London reported a lot of studies in the field of astrophysics and atmospheric sciences to improve spectroscopic database quality. ${ }^{8}$ The FTS technique has provided highly resolved and accurate spectra over the past decade but not below the beam splitter limit. For instance, Nave et al. ${ }^{9}$ achieved precision measurements of Fe II lines from 90 to $200 \mathrm{~nm}$. To this end, they used two different types of instruments: a FT spectrometer to study the [150-200] nm range and a high resolution normal incidence grating spectrograph to complete the spectra. At least one order of magnitude of resolving power has been lost between the two techniques. It is remarkable that the FTS techniques never extended deeply toward high energy, although similar high resolution and accurate data are equally or even more desired. Indeed the 8-20 eV range corresponds to very highly excited states such as Rydberg states (converging toward the different atomic or molecular ionizations and dissociation limits) or valenceband superexcited states. Their experimental study at ultimate resolution would not only be highly desirable for a detailed comparison with existing theoretical models but would have also, for small molecular systems, a wide range of applications in astrophysics (interstellar medium, planetary atmospheres) as well as terrestrial atmosphere (ionosphere in particular) and pollution studies. Note that in the VUV range, FTS can provide molecular dynamics (photochemistry) information since absorption is the initial step of a photochemical reaction leading to a shortening of the lifetime of the excited state in most case by (pre)dissociation. Such a process can be observed in absorption by a line broadening of the spectra.

There are very few projects involving amplitude division interferometers in the VUV. As a matter of fact, the major difficulty for beam splitter realization is related to the strong absorption of materials, which makes it very difficult to produce with a required flatness and over an extended spectral range. For example, the use of a $\mathrm{MgF}_{2}$ beam splitter determines the transmission limit of the London Imperial College Group FTS instrument $(\sim 140 \mathrm{~nm}) .{ }^{10}$ Manufacturing accurate beam splitters below this limit is a current research field, and two kinds of beam splitters are considered for integration 
into an interferometric system. The first one is based on multilayers deposited on very thin membranes. ${ }^{11}$ Besides the fact that multilayer beam splitters are difficult to manufacture, they are also strongly chromatic, which restricts the instrument to narrow band applications. ${ }^{12}$ Another important constraint is due to the lack of material with adequate optical constants for manufacturing multilayer beam splitters in some regions of the VUV spectrum. The other solution takes advantage of gratings acting as beam splitters but also with restriction to a specific bandwidth. ${ }^{13}$ A Mach-Zehnder arrangement has been developed using this technology in order to probe large scale laser created plasma. ${ }^{14}$ A slightly different idea was proposed by Howells et al. ${ }^{15}$ for a MachZehnder scanning interferometer consisting of a reflecting/ transmitting multislit arrangement. They designed and built a FTS based on this principle, with the aim of recording the absorption spectrum of $\mathrm{He}$ in the region of double ionization $(\lambda \sim 15 \mathrm{~nm})$, with a resolving power higher than $10^{6}$. A similar design was also studied theoretically for the VUV and tested in the visible by another group. ${ }^{16}$ Recently, a nice solution has been proposed using a high harmonic generation source. ${ }^{17}$ The basic idea is to produce two spatially separated and mutually coherent VUV pulses and let them interfere in the far field. One still have the same precision requirements, but the beam splitting problem is transferred to the infrared laser pump setup. As far as we know, these are the very few projects related to FT absorption spectroscopy, and none of them has given to date a wide band, flexible instrument for routine spectrometric experiments.

We believe that an amplitude division interferometer is not well adapted as a flexible instrument in the VUV range. We consider that another approach is left to overcome the beam splitter difficulty, namely, not to use any beam splitter at all. In other words, it consists of using a wave front division interferometer (WDI) instead of an amplitude division interferometer. Static WDI has already been implemented and used as a powerful tool in the VUV-extreme UV (EUV) range. ${ }^{18-22}$ The main difficulty of the present work resides in the design of a scanning WDI (instead of a static one) while fulfilling the specifications required by the FTS technique. Such a WDI-based FTS is aimed to be fed with photons emitted by an undulator from a synchrotron facility since this type of radiation is the only broadband, high brilliance light source in the VUV range.

In summary, we have designed such a FTS system operating in the VUV region $(\lambda \geq 60 \mathrm{~nm})$ with about a $5 \times 10^{5}$ theoretical resolving power (at the minimum working wavelength of $\lambda=60 \mathrm{~nm}$ ). In the next section, we describe its principle based on a modified Fresnel bimirror. Then, an original control system for accurate scanning will be presented in details followed by the overall optical implementation. The results of preliminary tests will be shown as well as the first spectrum obtained on a synchrotron beamline (SU5 beamline at Super-ACO): the Schumann-Runge absorption bands of $\mathrm{O}_{2}$ from 185 to $200 \mathrm{~nm}$. Finally, the end of this article is devoted to the instrument's recent progresses and future developments.

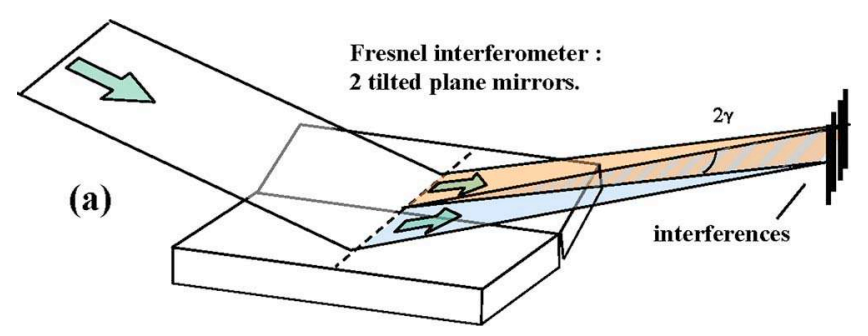

(b)

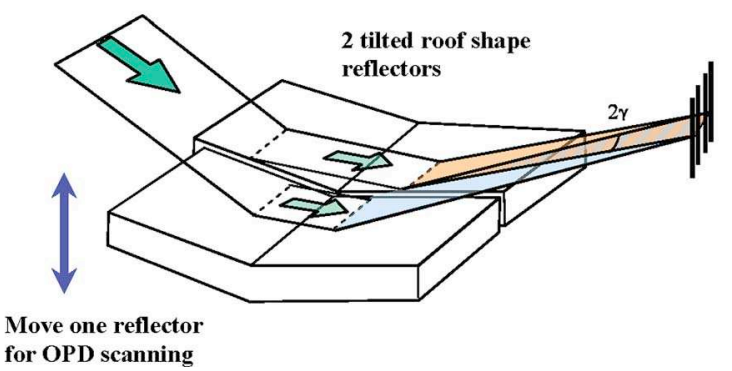

FIG. 1. (Color online) A WDI for FTS. (a) The simple Fresnel bimirror interferometer. This setup is not appropriate for a scanning WDI. (b) In order to keep the same two interfering rays during the scan, one needs to replace each mirror by a roof shaped reflector. The two roof reflectors are tilted so as to make the two beams interfere, producing a pattern of equidistant straight lines.

\section{THE VUV INTERFEROMETER OPTICAL SCHEME: PRINCIPLES AND DESIGN}

The Fresnel bimirror interferometer is one of the simplest WDIs, which involves only plane mirrors. It is basically a symmetrical setup, which means that both arms have symmetrical roles and identical design unlike the Lloyd's mirror, ${ }^{24}$ an even simpler design at first glance. As a direct consequence, it is quite easy to work within the zero OPD. The classical Fresnel bimirror scheme consists of two mirrors having a relative tilt with respect to each other in order to make the reflected half beams overlap. As mirrors are illuminated across the edge, two emerging half beams are generated and interfere downstream in the overlapping region ( $2 \gamma$ crossing angle) if suitable coherence conditions are fulfilled [Fig. 1(a)]. This generates a linear fringe pattern modulated by a Fresnel diffraction pattern from the common edge acting as two slit edges. One mirror can be moved for path difference scanning. More precisely, the "efficient" displacement vector is the component of the actual vector, which is perpendicular to the moving mirror surface. However, this configuration would not work well, as explained hereafter, and we use a modified configuration in which each mirror is replaced by a roof reflector as seen in Fig. 1(b).

This scheme guarantees that a particular interfering ray pair is issued during scanning from the same emitted ray pair, provided that the displacement vector is perpendicular to the roof edge and parallel to the roof bisector. In these conditions, no spurious time-modulation of the fringe can occur from spatial intensity modulation of the beam or from variations in spatial coherence conditions. ${ }^{23}$ In addition, the symmetry of arms (role and configuration) and their geometrical proximity allows to expect that any mechanical and thermal drifts of each arm relative to the whole setup will be very similar, and therefore they should produce a small dif- 
ferential drift, i.e., a limited perturbation of interferences ("common mode" rejection).

Roof reflectors with $90^{\circ}$ roof angle were used, i.e., $45^{\circ}$ for the incidence angles. With such a design, one has OPD variation $=2 \times$ displacement of the mobile roof reflector in the sense given above. For practical reasons related to implementation, the source and the detector stand at equal distance $D$ from the interferometer (this is neither mandatory nor critical).

At this point, it is necessary to make the choice between a symmetrical OPD scan, roughly from $-\Delta_{\max }$ to $+\Delta_{\max }$, and an asymmetrical OPD scan (from 0 to $\Delta_{\max }$ ), which provides in principle the same information content. As it is well known, the first choice requires the use of the exponential FT, while the cosine FT is used in the second case. The main advantage of the asymmetrical scan is to provide the same spectral resolution for half the scan range as compared to the symmetrical case. However, in this configuration, one needs to know accurately the origin of the path difference scale, i.e., the true zero path difference, which is not necessary when performing an exponential FT from a symmetrical scan. Owing to the difficulty of implementation of a large scanning range with the required accuracy (see Sec. III), we considered that the reduction in range provided by asymmetrical scan is of primary interest, and we chose therefore to use asymmetrical scan and cosine FT. The need for zero path difference determination will be addressed later in Sec. $\mathrm{V}$. With this configuration, a $7.5 \mathrm{~mm}$ displacement in the mobile roof reflector yields a $15 \mathrm{~mm}$ maximum OPD and a $5 \times 10^{5}$ resolving power at $\lambda=60 \mathrm{~nm}$.

It should be noted that as all WDI, the bimirror scheme cannot be adjusted for a flat interference state; the state of interference is not invariant across the field because the wave vectors of the interfering waves are not collinear, thus generating linear fringes in the detector plane. This is responsible for the partial (one dimension) loss of the "Jacquinot (étendue) advantage" because the interference signal can only be integrated along the fringe direction. More precisely, sampling the state of interference during the scan implies that the detector size (perpendicular to fringe) should be smaller than the smallest fringe spacing, typically from $1 / 4$ to $1 / 3$, in order to keep a correct signal contrast. However, to optimize the photometric detection, a large detector is desirable to collect as many photons as possible. This means having an as large fringe spacing as possible, i.e., a small crossing angle. Obviously, the limit is set by the fact that the two half beams must still overlap. In other words, the optimal geometry is reached by roughly equating the fringe spacing and the beam overlap in the detector plane through the beam crossing angle $2 \gamma$. This leads to an approximate relation between the mirror tilt $\gamma$, the smallest wavelength $\lambda$ to be used, and the distance $D$, namely, $2 \gamma D \cong 2(\lambda / 2 \gamma)$ considering that the source and the detector are located at equal distances from the reflectors. $D$ is set by a practical constraint on the SU5 Super-ACO synchrotron beamline where the instrument was implemented $(D=0.8 \mathrm{~m})$; therefore the only free parameter left is $\gamma$. Considering the nominal shortest working wavelength to be $\lambda=60 \mathrm{~nm}$, this yields, for a first estimate of the tilt angle,

$$
\gamma_{\mathrm{opt}} \approx \sqrt{\frac{\lambda}{2 D}}=0.19 \mathrm{mrad}
$$

We will now consider the more realistic situation of an extended spatially incoherent, spectrally narrow source. It is well known that by increasing the width of the source slit, we obtain more light, but simultaneously, the fringes become less and less distinct. In order to make observable the interference between the two beams produced by an extended source, an important condition must be fulfilled: we need a certain amount of spatial coherence (one dimensional in the case of the Fresnel interferometer). Usually, the available degree of coherence can be estimated using the Van CittertZernike (VCZ) theorem: the degree of coherence on a screen illuminated by an extended quasimonochromatic incoherent source is the absolute value of the normalized FT of the intensity function of the source. ${ }^{24}$ Note that in the case of synchrotron radiation (particularly for an undulator source), the beam is known to be partially coherent throughout the beamline. It has been demonstrated that the VCZ theorem may underestimate somehow the degree of coherence on the image source in the case of a VUV/soft x-ray undulator source. ${ }^{25}$ However, we suppose that we are in the worst conditions, considering an incoherent source at the SU5 undulator beamline endstation. The high spectral brilliance of modern synchrotron sources provides the required coherent flux at the experimental station. Thus, according to the VCZ theorem we get a simple relation between the source slit width $s$ and the tilt angle $\gamma$ by setting, for example, a degree of coherence higher than 0.6 , namely, $s \leq \lambda / 2 \gamma$. For $\gamma=\gamma_{\text {opt }}$ $=0.19 \mathrm{mrad}$ and $\lambda=60 \mathrm{~nm}$ the maximum slit width is $150 \mu \mathrm{m}$ in the VCZ approximation.

To improve this first estimation, we have simulated numerically the interferogram detection, assuming the shortest wavelength to be used, i.e., $\lambda=60 \mathrm{~nm}$. To this end, we maximized either the detected signal alone (interference modulation) or the detected interference signal to photon noise ratio, the free parameter being the angle $\gamma$. Constraints are the detector size (a constant fraction of the fringe spacing from $1 / 3$ to $1 / 4$ ) and the spatial degree of coherence in the detector plane ( 0.6 considering a quasiincoherent source). This study includes the modulation of the fringe pattern by the Fresnel diffraction due to the mirror edges, which affects the interference field particularly near the zero path difference. Although the theoretical Fresnel bimirror introduces diffraction by one common mirror edge, here, there are four mirrors (two in each arm), and the roof reflectors must be physically separated from each other to allow for a safe scan. We set the gap between the two reflectors to $100 \mu \mathrm{m}$. It comes out of the simulations that the optimization curve presents a rather flat maximum for $\gamma$ between 0.4 and $0.5 \mathrm{mrad}$. For practical reasons, we choose $\gamma=0.43 \mathrm{mrad}$ as our final tilt angle optimized value. Assuming equal source slit and detector to interferometer distances $D(D=800 \mathrm{~mm})$, the fringe spacing is $150 \mu \mathrm{m}$ at $\lambda=60 \mathrm{~nm}$. In order to keep the degree of coherence above 0.6 , the slit width should not exceed $70 \mu \mathrm{m}$ for $\lambda=60 \mathrm{~nm}(120 \mu \mathrm{m}$ for $100 \mathrm{~nm})$ with $\gamma$ 
$=0.43 \mathrm{mrad}$. One single horizontal slit is located in front of the detector, whose size $(50 \mu \mathrm{m})$ is adapted to the smallest fringe spacing we are planning to manage.

To implement this interferometer in the context of efficient FTS, two classes of problems must be solved. (1) The optical system must meet very tight specifications and keep them during scanning and on the long term; this is a matter of fine optical and mechanical design. (2) The OPD must be known during scanning at a very high level of accuracy, typically $1 / 15$ of the smallest wavelength to manage, i.e., at a nanometric level for a centimetric scanning range. This is by far the main challenge to address.

\section{A CONTROL SYSTEM FOR ACCURATE INTERFEROGRAM SAMPLING}

Our goal is to set up the scanning interferometer described in Sec. II, associated to a suitable sampling system, so as to meet the conditions required for $5 \times 10^{5}$ resolution at the minimum wavelength of $\lambda_{\min }=60 \mathrm{~nm}$. As it is well known, the key point is to provide a constant sampling interval of the interferogram, in terms of OPD, with tolerances scaled by the shortest wavelength in the spectrum. The required accuracy of the sampling positions can be derived from classical FTS error analysis; ${ }^{2}$ to obtain undistorted spectra at wavelength $\lambda$, the average error on sampling positions (OPD) with respect to a regular sampling must be smaller than $\lambda / 15 \mathrm{rms}$. This amounts to $4 \mathrm{~nm}$ rms (OPD) over the full sampling range for $\lambda=60 \mathrm{~nm}$, i.e., a $7.5 \mathrm{~mm}$ translation.

The most straightforward solution would be the design of a high quality translation stage in order to provide the required regular translation of the moving reflector, i.e., free of unwanted speed fluctuations, but also of unwanted rotation, that could perturb the interference state. However, we considered that no mechanical device can by itself reach such a specification without unreasonable effort. Therefore our strategy lies on (i) the use of a "good" translation stage associated to a linear actuator and (ii) the association of this mechanical basis to some dedicated control system, so as to measure continuously the OPD variations in order to determine the sampling positions and to correct for angular errors of the moving reflector. With this approach, the essential requirement for the mechanical basis is that it should produce a reproducible and fairly "smooth" trajectory free of vibrations or friction and with a good stability. On the contrary, its intrinsic trajectory errors (mainly departure from linearity and spurious rotations) can be those of good standard products, i.e., two orders of magnitude larger than required at the reflector's level.

The design and realization of this control or scanning system were indeed the real challenge of this project. To help solving the problem, we added two additional rules. (1) Scanning must be continuous rather than a by stop-and-go (step by step) between the successive sampling positions. Therefore sampling should be performed "on the fly," upon triggering by the control system. (2) One must probe directly the relative displacement of both interferometer arms as close as possible to the WDI reflecting surfaces, so as to (a)

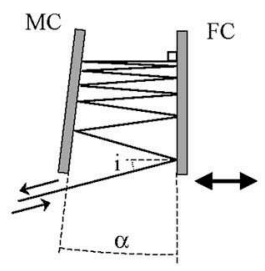

(c)

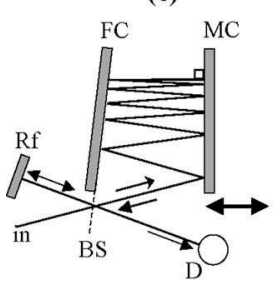

(b)

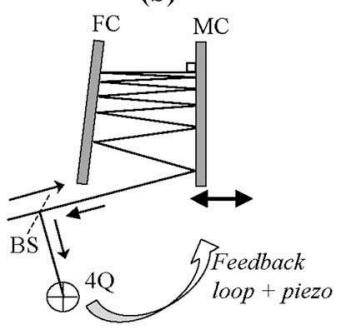

(d)

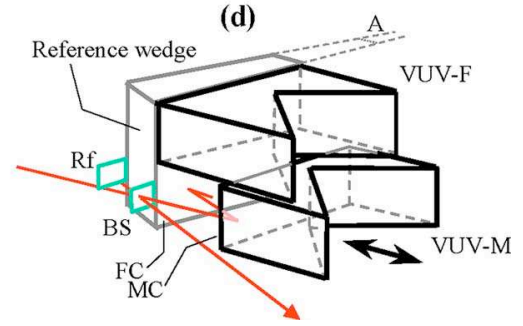

FIG. 2. (Color online) The scanning control system. (a) Principle of the multireflection system. (b) Deflectometer for angular stabilization of the moving reflector. (c) Interferometer for high sensitivity determination of the displacement of the moving reflector with respect to the still one. (d) Implementation into the VUV scanning interferometer. FC and MC stand respectively for fixed and moving control mirrors; BS, beam splitter; 4Q, fourquadrant detector; Rf, reference arm mirror of the control Michelson interferometer; D, control interference detector; and VUV-F and VUV-M, fixed and moving reflectors of the VUV interferometer, respectively.

reject common mode drifts with respect to the rest of the setup. If the probed path and the actual VUV path are not superimposed (which will be the case for practical reason), it must be checked that no differential error will arise between the probed and the actual path differences, e.g., from spurious angular deviations of the moving arm during scanning. Besides, the link between the VUV reflectors and the control system probe must be a solid part, as free as possible of vibrations and thermal expansion. This leads to a design consisting of compact, solid optical blocks, (one for each arm, including the control system) made of low thermal expansion materials such as silica or even Zerodur.

\section{A. Principle of the control system}

Owing to the required motion high accuracy, we probe during the scan both the translation and the angular jitter of the moving reflector by using a single optical system based on a multireflection scheme of a reference external visible laser. To make its principle clear, let us consider two vertical plane mirrors making an angle $\alpha$, both mirrors being perpendicular to the plane in Fig. 2. The geometrical intersection of these two mirrors is therefore a virtual vertical line perpendicular to the figure plane. One mirror is still and the other one is moving perpendicular to its surface. The following analysis is made for rays all lying in the horizontal plane: extension to deviations in the vertical plane is straightforward and gives essentially the same results. As sketched on the figure, we illuminate the mobile mirror in the horizontal plane by a laser beam (plane or weakly spherical wave) with an incidence angle $i$. Generally, a multiple reflection system (the "fan") takes place between the two mirrors. When the condition $i=(p-1) \alpha$ ( $p$ is any integer greater than one) is 
fulfilled, the beam comes back exactly superimposed on itself and finally comes out of the two-mirror system [Fig. $2(\mathrm{a})$. We call this situation "resonance," and "resonance condition" is the associated equation. Note that the "folding back" reflection occurs on either mirror, depending on the parity of $p$. Parameter $p$ can be understood as the number of spots present on the reflecting surfaces of the fan, all but one being a double reflection, one forward and one backward.

This scheme allows a twofold control of the moving mirror. First, by probing the return beam with the help of a four-quadrant (4Q) photodiode suitably aligned with the incoming beam axis, one gets a very sensitive deflectometer (two directions), which opens the possibility of a looped feedback for a two dimensions angular stabilization of the moving reflector [Fig. 2(b)]. Second, if associated, by means of a beam splitter with a fixed reference arm, one obtains a Michelson interferometer [Fig. 2(c)], which provides a very sensitive interferometric displacement probe due to the multireflection scheme. This latter design was successfully used by Chandra and Rohde, ${ }^{26}$ who were able to reach a linear resolution as low as $10 \mathrm{~nm}$ for the measure of a mirror displacement.

Obviously, these mirrors must be part of two optical blocks, respectively, containing the still and the moving VUV reflector. We have considered that it is not convenient to implement the high sensitivity control system on the VUV side of the reflectors. Instead, we use the rear surface of the moving VUV reflector as the moving mirror of the control fan. The still block is arranged so as to get the second (still) mirror of the control fan facing the first one at the desired angle. Also it contains the beam splitter and the reference mirror, the angle of which is defined by a solid glass prism (the "reference wedge"). For thermal stability, all blocks were made of Zerodur or silica. This is illustrated by the scheme in Fig. 2(d).

Then, the principle of operation consists of having simultaneously both controls (deflectometer and interferometer, fed by the same laser beam) properly adjusted. When both conditions are fulfilled (for a given $p$ ), the angular positions of all surfaces are uniquely defined with respect to the control beam due to the constraint added to the resonant fan by the aligned interferometer. As a matter fact, the geometry depends on two parameters only, the reference wedge angle $A$ and the integer $p$.

First, to get the interferometer properly aligned, the reference mirror must be in autocollimation condition, which implies that the control beam is incident onto the beam splitter with angle $A$. Note that this does not depend on $p$. Then, the incidence angle $i$ onto the moving control reflector is $A$ $-\alpha$ ( $\alpha$ is the angle between the fan's mirrors). Introducing the resonance condition $i=(p-1) \alpha$ yields $A-\alpha=(p-1) \alpha$, that is, $\alpha=A / p$ and $i=(1-1 / p) A$. In other words the incidence and fan angles are fixed by one solid optical part only, the (beam splitter-reference) mirror block. As this block is not a moving part, it can be extremely stable and well known. This should guarantee the stability and reproducibility of the system to a high degree of accuracy. Moreover, it defines in some sense an "absolute" angular position of the moving reflector with respect to the rest of the setup.
As for the vertical plane, it is intuitive that any spurious rotation of the moving mirror around a horizontal axis would produce a vertical deviation of the return beam of the deflectometer with exactly the same sensitivity as in the horizontal plane. In fact equations are the same, except for the vertical incidence $i$, which is zero and should stay zero when the system is locked.

The arrangement depicted in Fig. 2(d) has an important consequence regarding the required angular stability during the scanning. Because the probing region of the control interferometer onto the moving reflector block is spatially separated from the VUV side, residual angular errors of the moving block can yield differences between the translation as measured by the control system and the actual OPD, as seen by the VUV interferometer. A thorough stability analysis of the whole optomechanical system shows that to guarantee tolerable sampling errors, the angular stability of the moving reflector must be less than $1 \mu \mathrm{rad}$, i.e., a tighter specification than required for fringe pattern stability (a few microradians ${ }^{27}$ ).

\section{B. Probing the scanning translation}

As explained in the previous section, we are using a Michelson interferometer configuration with the mobile arm made of the two-mirror wedge arrangement [Fig. 2(c)]. As a two-wave interferometer, it provides a sinusoidal interferometric signal that allows the accurate monitoring of the differential displacement of the interferometer moving arm with respect to the still one. Owing to the multireflection amplification, the period of the signal is roughly inversely proportional to the number of reflections $p$, namely, period $=\lambda_{\text {laser }} / 2 p^{\prime}$, with $p^{\prime}=C p$, where $C$ is a geometrical factor, which is close to and less than one and depends on $p$ and $A$. Note that this is the period in terms of the displacement of the moving reflector.

If the VUV acquisition is triggered by this signal at each half-fringe, one gets a sampling interval equal to $\lambda_{\text {laser }} / 4 p^{\prime}$ (displacement), i.e., $\lambda_{\text {laser }} / 2 p^{\prime}$ (OPD), which sets the Nyquist limit $\lambda_{\text {min }}$ for broadband spectra to $\approx \lambda_{\text {laser }} / p^{\prime}$. One clearly sees here the interest of the $p$-folding geometry allowing to transfer in the VUV range the Nyquist limit. With $\lambda_{\text {laser }}$ $=633 \mathrm{~nm}$, choosing $\alpha=1^{\circ}, i=10^{\circ}$, and $p=11$ as the basic configuration yields $p^{\prime} \approx 10.934$, and the sampling interval is $\lambda_{\text {laser }} / 2 p^{\prime}=29 \mathrm{~nm}$ in terms of OPD, setting $\lambda_{\text {min }}$ to $58 \mathrm{~nm}$. It is worth noting that this sensitivity is obtained by dividing the sinusoidal period by two, which is a very simple processing and gives accurate results. On the contrary, getting the same sensitivity from classical commercial interferometers would require a large electronic subdivision of each fringe, thus increasing the risk of sampling errors (or requesting for an accurately constant scanning speed). Also, the interferometric indexation system provides in principle a perfect rejection of the speed fluctuations of the moving reflector.

Because $p$ is a free parameter, the wavelength range limit can be moved toward shorter (longer) wavelengths by increasing (decreasing) $p$, the number of reflections. Tilting the moving reflector by $\Delta i$ until a new alignment is obtained leads to $q=p \pm 1$. According to Sec. III A, one has $i=(1$ $-1 / p) A$ and $i^{\prime}=[1-1 /(p \pm 1)] A$, which gives $\Delta i$ 
$= \pm A /[p(p \pm 1)]$. Accordingly, the fan angle $\alpha$ changes into $\alpha^{\prime}=A /(p \pm 1)$. This feature gives the ability to control the sampling interferogram period or, in other words, to adapt the Nyquist frequency to the spectrum being recorded and therefore its resolution. This possibility was not integrated in the actual prototype as an in-vacuum adjustment but scheduled for the next ultrahigh vacuum (UHV) instrument (see Sec. VII).

\section{Probing angular stability}

As mentioned, the same multireflection system provides a sensitive $2 \mathrm{D}$ deflectometry (pitch and yaw with respect to the translation direction). A beam splitter is positioned on the laser beam path in order to isolate and analyze the reflected beam with the help of a $4 \mathrm{Q}$ photodiode. It is important to place the 4Q photodiode axis accurately on the retroreflected axis [Fig. 2(b)] in order to avoid a systematic angular deviation upon scanning. Angular stabilization is obtained by including the 4Q photodiode sensor and suitable piezoactuators in a feedback loop to correct for the moving reflector angular deviations.

Basically, the system converts the angular deviation of the reflector into a position deviation on the photodiode by way of an amplification factor $A$, a function of the number of reflections $p$ and of the geometrical distances involved. Our standard configuration $\left(\alpha=1^{\circ}, i=10^{\circ}\right.$, and $\left.p=11\right)$ gives 7.7 $<A<10 \mu \mathrm{m} / \mu \mathrm{rad}$. $A$ is not constant during a scan because this parameter is related to the distance between the two plane mirrors, which is variable by principle. When the loop is closed, the VUV moving reflector is locked on a well defined direction with an accuracy limited by the multireflection device sensitivity, the fan optical surfaces accuracy, and the feedback loop noise. Ultimately, this noise gives the equivalent tilt error correction limit on the moving mirror. It is worth mentioning that the angular jitter of the probing laser is not amplified by the multireflection scheme. This is equivalent to having a rejection of the laser beam angular fluctuations with respect to the mirror angular changes. The measured tilt error correction limit due to noise is approximately $0.15 \mu \mathrm{rad}(\mathrm{rms})$ for $p=11{ }^{27}$ This is compatible with the stability required to guarantee an accurate determination of the VUV OPD (see above).

\section{Physical limitations of the scanning}

Obviously, the ray-fan transversal dimension increases when the gap between the control mirrors increases. Therefore, the finite length of the optics sets an upper limit to $p$, given the desired travel of the moving reflector, i.e., ultimately the spectral resolution. However, the actual limit depends on what type of resolution is considered. At constant interval between spectral samples, i.e., for a given scanning range, the size of the ray fan increases quadratically with $p$. However, at constant resolving power (i.e., constant number of samples), the size of the fan also increases, but much slower, because the distance between interferogram samples becomes smaller when $p$ increases. As an example, for the standard set $(\alpha=1, i=10$, and $p=11)$, the fan size is $31.4 \mathrm{~mm}$ for $1 \mathrm{~K}^{2}$ samples $(1 \mathrm{~K}=1024)$. For $p=16$, it would be 38 $\mathrm{mm}$ at constant sample number but $\approx 46 \mathrm{~mm}$ at constant scanning range. Giving the basic set of parameters $(\alpha=1, i$ $=10$, and $p=11$ ), we choose $50 \mathrm{~mm}$ wide fan mirrors, which would give a maximum resolving power of 500000 at $60 \mathrm{~nm}$.

\section{IMPLEMENTATION AND REALIZATION}

A first important requirement for VUV operation should be kept in mind: the system must be operated in a clean vacuum environment, owing to the high VUV flux density illuminating the VUV reflectors. This is true for all parts, including driving motors, some electronics (as few as possible), detectors, except the control laser, which must be kept in air, due to its technology. This requirement is indeed an issue for the mechanical design and the selection of elements such as motors.

\section{A. Optical blocks}

In order to meet the desired stability conditions, the optical blocks are made of assembled elementary parts, flats, prisms, and one beam splitter. Assembly is made by optical gluing in such a way to respect the parallelism of assembled surfaces. For practical reasons, we decided to set the mirrors normal vector into a horizontal plane. Although this is not an issue, the overall mechanical balance of such a scheme seems better compared to the situation with the normal vector into a vertical plane. This configuration leads to an important point concerning the polarization of the source. In order to optimize the reflected flux we shall use the $S$ polarization of light, corresponding to the vertical linear polarization (the only one that allows a significant transmission in the VUV). In addition, the overall reflection of the interferometer must be fairly efficient from 200 to $60 \mathrm{~nm}$. To improve reflectivity, a thin platinum coating was deposited on the mirrors. Platinum was chosen because it is probably one of the best metallic layer candidates, as it does not suffer oxidization and has a fairly high and flat reflectivity over the desired VUV spectrum region. From complex indices tables $^{28}$ and using the Fresnel equations, we determined the interferometer overall transmission (two consecutive $45^{\circ}$ incident reflections) to be around $10 \%$ in the $60-200 \mathrm{~nm}$ range for $S$ polarized light.

\section{B. General implementation}

The scheme was implemented as depicted in Fig. 3. A monomode, $f$-stabilized $\mathrm{He}-\mathrm{Ne}$ laser (Spectra Physics model 117A) is first carefully aligned with the translation vector of a good off-the-shelf translation stage (Newport M-UMR8.51), double ball bearing, prepared for high vacuum operation with $50 \mathrm{~mm}$ range. Then, it is necessary to separate the indexation and the deflectometric channels because upon displacement of the moving arm, the interferometric control signal follows a sine function and the tilt correction by means of the four quadrants photodiode requires a dc signal. Moreover, optimal focusing conditions are not the same for both channels. Therefore, a beam separator is installed to obtain two strictly parallel beams vertically separated by $14 \mathrm{~mm}$. The top beam is for the control interferom- 


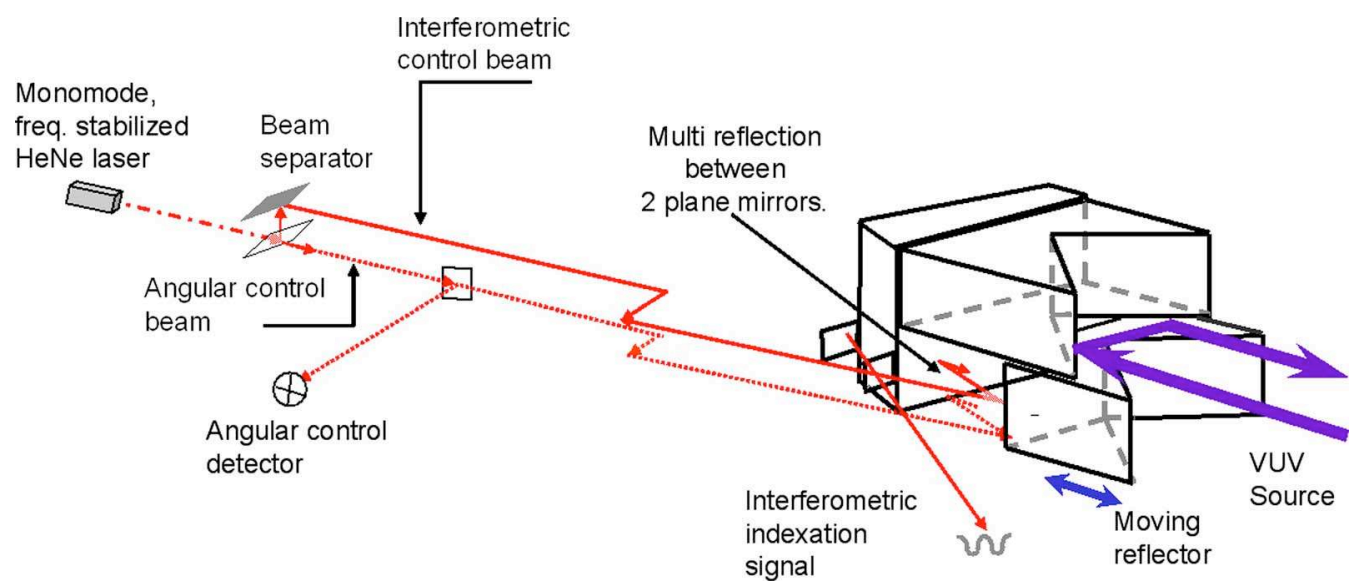

FIG. 3. (Color online) Scheme of the VUV FT spectrometer setup. On the front side, the WDI faces the VUV synchrotron beam. The angular control and measurement displacement setup is shown on the back side. The incident HeNe laser beam is split to separate the interferometric indexation control system (upper beam) and the deflectometer for the tilt control (lower beam). Both systems are based on the multireflection scheme depicted in Fig. 2.

eter, while the bottom beam is for the deflectometer. Then, although only the deflectometer is concerned, for symmetry reasons both beams cross a silica plate, which is coated on its lower half, to act as a beam splitter for the return path of the deflectometer toward the $4 \mathrm{Q}$ detector. After, both control beams are redirected by a two-mirror assembly to produce a deviation equal to the nominal incidence angle at $p=11$ so that the moving control mirror, once the system aligned, is perpendicular to the stage translation vector. Finally, the two optical blocks are inserted. The upper block is the still one. For the initial static alignment, it can be adjusted vertically and angularly by means of micrometric screws, two of them being high sensitivity, externally controlled piezoelectric screws (New Focus Picomotor). The lower block is the moving one. It is attached to the translation stage through a twoaxis adjustable mount (one vertical and one transverse horizontal) based on standard friction-free flexural pivot. This allows to dynamically adjust during scan the pitch and yaw of the moving optical block. This is made by piezoactuators driven by a servo-loop, which locks the deflectometer signal to zero. The "gap" between optical blocks, i.e., the vertical separation between the two reflector blocks, is set to $100-150 \mu \mathrm{m}$.

The translation stage is driven by a dc-motor pushing actuator (high vacuum compatible). Therefore, the control signal is frequency-modulated according to speed fluctuations as well as the VUV signal from each pure wavelength. These fluctuations are cancelled, thanks to the very principle of the control system, which uses directly the interferometric signal to generate the sampling signal instead of the time. However, care must be taken not to introduce time shifts (i.e., sample timing errors) between the control signal and the VUV signal through phase rotation due to the amplifier bandpass. Analysis shows that by matching the frequency response of all concerned amplifiers, no significant differential phase-shift is introduced in both channels even when the signal frequencies reach the amplifier's cutoff. The system general synoptic and internal links are summarized in Fig. 4.

\section{System alignment}

As indicated in Sec. III C, the 4Q diode (deflectometer detector) must be positioned on the laser beam direction axis (in fact its image is given by the beam splitter). In principle, one should set the diode position with a precision related to the 4Q diode sensitivity $(\sim 1 \mu \mathrm{m})$. In order to reach this value, we use a simple but accurate adjustment procedure based on the reflection of the laser onto a liquid mirror. This horizontal reflecting surface is used as a movable datum plane in a vertical axis; the reference is gravity. The object of this adjustment is to superimpose the incident beam, the reflected beam, and the center of the diode for two different positions of the liquid mirror.

Then, the main adjustment consists of producing simultaneously the resonance condition in the optical fan and a flat field condition in the control interferometer. What would appear as a difficult task is actually made easier by the geometry of the optical blocks and the incident beam, owing to the constraints they impose. The first step is to place the reference mirror of the control interferometer (which is a part of the still block) in autocollimation with the incident beam (i.e., coming back on itself). This is obviously a part of the whole process, which can be done without the moving block in place. Once done, there is only one way to introduce the moving reflector and fulfill a given resonance condition (one

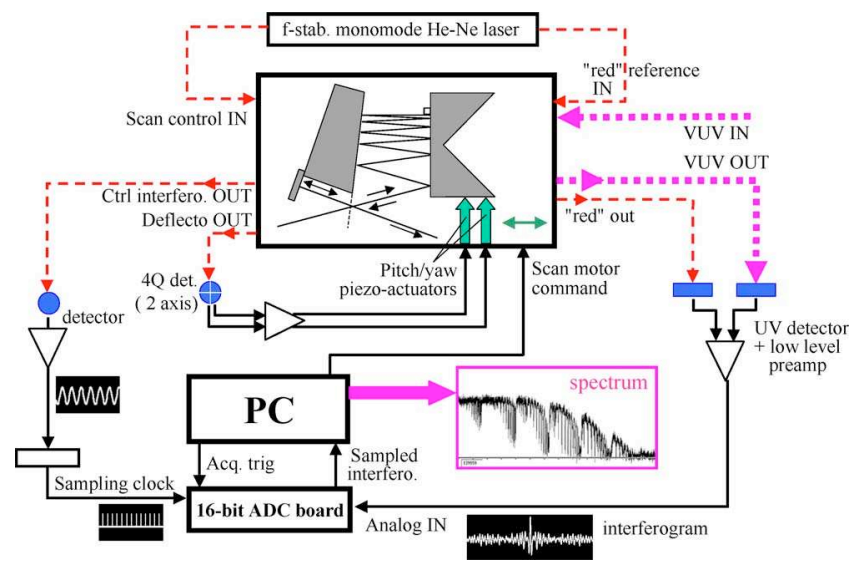

FIG. 4. (Color online) Synoptic of the FT spectrometer system (see text for details). Dotted lines indicate photon beams (red He-Ne or VUV). Note that all photons beams and detectors are in the vacuum, except for the source beam ( $f$-stabilized laser), which is injected into the vacuum through an optical window. All electronics but the detectors are in air. 
$p$ value). In other words, once the deflectometer is adjusted, the flat interferometric field is automatically obtained. In practice, it is quite difficult to obtain directly the perfect position of the still block, but the first step adjustment is generally good enough to allow the servo-loop of the deflectometer to lock up and the control interferometer to show some modulation due to the residual vibrations of the system (note that one interference half-period is produced when the control mirrors move only by $15 \mathrm{~nm}$ relative to each other). Then, it is only necessary to maximize this modulation by adjusting the still block while keeping the deflectometer loop locked. In theory, we can choose any number of reflections $p$ starting from the basic configuration $\left(\alpha=1^{\circ}, i=10^{\circ}\right.$, and $p=11$ ), with the limitations set by the control mirror sizes.

\section{Interference detection}

The VUV detection system consists of an IRD VUV silicon array of three photodiodes, $1 \times 1 \mathrm{~mm}^{2}$ (model AXUV-3EL manufactured by International Radiation Detectors, Inc.), associated with a $50 \mu \mathrm{m}$ slit, to restrict the detected area to $1 / 3$ fringe at most (see Sec. II). Only the central diode is used to detect the VUV interference; the two others are provision for normalization of possible source fluctuations. Also, an array of input horizontal slits is inserted on the input side, at the focusing point of the SR beam, to restrict the source vertical size for spatial coherence enhancement if necessary. Four sizes are available, namely, 50, 100, 200, and $400 \mu \mathrm{m}$.

\section{RECORDING AND PROCESSING INTERFEROGRAMS}

As we just mentioned, the control interferometer is not free of vibrations. However, the system was designed under the implicit assumption that the travel direction never reverses during scan. Obviously, this is not realized if the average traveling speed is smaller than the average vibrational speed and the sampling quality will be impaired. The solution to this problem relies on using a large enough average scanning speed and sampling on the fly, each time the control interferometer crosses its average value, i.e., one sample every one half-period of the control interferometer.

Experience shows that the minimum translation speeds range from $40 \mu \mathrm{m} / \mathrm{s}$ (very quiet conditions, special table) to 150 or even $200 \mu \mathrm{m} / \mathrm{s}$ (busy synchrotron experimental hall with close source of vibrations, essentially through the ground). In any case, the effect of too large a vibration level is to add spurious samples, which to some extent, can be detected and corrected for (see below).

\section{A. Acquisition of the VUV interferogram and interferogram processing}

The VUV detection diode is followed by a low-noise instrumentation op-amp, converting the diode current into voltage through a $50 \mathrm{M} \Omega$ resistance. No significant noise is added to the signal at this stage. Then, the signal is digitized by a personal computer (PC) analogic to digital converter (ADC) 16-bit board (Keithley model KPCI-3108). The control of acquisition clocking is not performed by the $\mathrm{PC}$ but merely by the board itself (i.e., hardware), which uses directly the signal from the control interferometer as the sampling signal through a dedicated "external clock" input. Such an arrangement is mandatory if one wants to perform a perfectly accurate sampling with a temporally irregular sampling rate (which is the case here). Samples are written directly into PC memory, as the ADC board takes control of the PC bus through bus mastering. Therefore, there is virtually no limit to the size of interferogram arrays to be acquired.

The heart of interferogram processing is the application of a discrete cosine FT to the set of interferometric sample. This should produce accurate spectra in terms of both the spectral scale and the line shape, but this is impaired by the fact that the sample set may be not perfect for various reasons. These are mainly the origin problem, common to all cosine FT based systems, the sampling errors (possible extra samples and sampling time errors), and the limited accuracy to the knowledge of the average OPD sampling interval, which affects the absolute calibration of the spectral scale. The first two issues are addressed hereafter. As for the absolute spectral calibration we did not focus on this issue yet. The experimental data show a typical relative accuracy in the $10^{-5}$ range (see Sec. VI).

\section{The origin problem}

As it is well known, failure to register a sample at the zero path difference brings a linear phase error into the spectrum when computed by exponential FT and a severe distortion of the ideal sinc impulse response when computed by cosine FT. In order to overcome this problem, we use the classical origin correction procedure due to Forman et al. ${ }^{29}$ The principle is to record a small symmetrical interferogram with a limited travel (i.e., limited resolution) and to compute a low resolution complex spectrum in order to extract the origin shift from the phase information. A correcting function (truncated, shifted sinc function) is then convolved with the full noncorrected interferogram in order to shift the sample set so that the first sample is relative to the zero OPD. This is implemented by starting the scan about 20000 samples before the zero OPD estimated position so that a $32 \mathrm{~K}$ symmetrical exponential FT is always possible.

\section{Checking and correcting for sampling errors}

As it was reminded before, the critical condition to get a good spectrum, i.e., a spectral impulse response as close as possible to the theoretical sinc function, is that the sampling comb is as regular as possible in terms of OPD of the VUV interferometer. Owing to the particular context of this instrument, i.e., the very small wavelength involved, and the lack of similar experiences (and of high resolution spectral data in the VUV range), we found necessary to introduce some checking of the "sampling error profile." Fortunately, this is quite easy to realize by using the $f$-stabilized control laser, namely, feeding the VUV interferometer with a small (10\%) part of the laser beam. This yields a "red interferogram," which is electronically added to the VUV interferogram and is sampled exactly in the same way. As a matter of fact, as 
far as a single frequency source is fed into the VUV interferometer, it should produce a perfect cosine interferogram, and any sampling position error acts as a phase error. Therefore, one can retrieve this error term by processing the actual interferogram for phase demodulation.

To this end, the exponential FT of one asymmetrical interferogram ( $N$ samples) is produced. Here the origin problem is of no concern because the interferogram results in one single line, with a width small enough to produce a negligible amplitude modulation along the scan. Besides, the detected modulation can be adjusted to be several orders of magnitude above detection noises. Then, the spectrum is processed in three steps in order to (1) isolate the spectral peak on a domain of $\pm n$ spectral samples (we use $n=256$ for a $N=1024$ K sample set), (2) shift this domain so that the peak is at or close to the spectral origin and fill in with zero to obtain a $2 N$-sample symmetrical spectrum, and (3) perform an exponential back Fourier transformation. This produces a quasipure cosine interferogram, the phase of which should be linear with the OPD variation or zero if the spectral peak is perfectly centered on the spectral origin. Any departure from linearity reflects the sampling error profile of the initial $N$-sample set. Due to the initial spectral filtering (first step above), this profile is averaged by a sinc window with first zero half width equals $N / 2 n$ samples (for $N$ $=1024 \mathrm{~K}$ and $n=256 ; N / n=2048)$. More precisely, the local sampling error profile along a scan $E(x)$ is computed from the phase error profile $\phi(x)$, possibly after some phase unwrapping, by the classical expression

$$
E(x) / T=\operatorname{Arctan}[\phi(x)] / 2 \pi .
$$

Here, $T$ refers to the reference interferogram period; a sampling error of one period yields a phase error of $2 \pi$. For convenience we use the sample interval as unit for the determination of $E(x)$.

Such an averaged error profile can be corrected by a locally constant interpolation using exactly the same algorithm as used for origin retrieval, producing a corrected interferogram with long-range sampling errors negligible over the whole spectral range. Note that it does not replace the origin retrieval process, which must be performed separately.

Obviously, this process does not work for sampling errors with short-range correlation, i.e., below $N / 2 n$ samples, typically a few samples to a few hundreds. However, the spectra calculated from corrected interferograms show that residual short-range errors do not degrade significantly the spectra. Note that the presence of such short-range residual sampling errors can be checked by subtracting the corrected interferogram from the raw interferogram.

\section{B. Testing of the scanning interferometer}

The very first spectrum that we produced with the present FT spectrometer was the visible-to-near UV emission spectrum of a mercury arc $(150 \mathrm{~W}$, high luminance), showing the self-absorption lines superimposed on broad emission lines. ${ }^{30}$ It was obtained in one single pass, with the nominal sampling interval of $29 \mathrm{~nm}$ (OPD) and $256 \mathrm{~K}$ samples, i.e., an absolute resolution of $0.66 \mathrm{~cm}^{-1}$. The scanning speed was $30 \mu \mathrm{m} / \mathrm{s}$, i.e., $2 \mathrm{~K}$ sample/s. To our knowledge, it was the very first FT spectrum ever obtained with this kind of scanning interferometer. Then, the instrument was systematically tested by studying the response to a single frequency, namely, the frequency-stabilized control laser, whose frequency is known and stable, better than $10^{-8}$ on the medium term (hours). As far as a single frequency source is used, the resulting interferogram should be a pure cosine (+dc level), and the corresponding spectrum is the impulse response of the instrument, the classical sinc function. Any departure should be considered as errors. Two aspects are to be considered, namely, the sampling position error and its profile as a function of the scanning position, and the spectral noise as a function of the wavenumber. In all cases, interferograms were recorded with the VUV sampling rate, i.e., one sample per $29 \mathrm{~nm}$ OPD change, which yields $\approx 21$ samples per laser fringe. $512 \mathrm{~K}$ samples were recorded, which give a spectral sample interval of $\delta \sigma=0.33 \mathrm{~cm}^{-1}$ with a cosine FT.

\section{Sampling error profile}

As we have implemented the sampling error profile extraction in the spectrum calculation software (see Sec. V A), we were able to check profiles for each interferogram recorded with the He-Ne control laser as a source. The rms departure from linearity was generally within 0.15 times the sampling interval, i.e., from 4 to $5 \mathrm{~nm} \mathrm{rms} \mathrm{(OPD).} \mathrm{The} \mathrm{im-}$ portant result is that the error is essentially systematic, i.e., reproducible from scan to scan. ${ }^{31}$ Therefore the major part can be corrected by a locally constant interpolation using exactly the same algorithm as used for origin retrieval, producing a corrected interferogram with sampling errors negligible over the whole spectral range. We have checked the efficiency of the correcting process on strongly distorted interferograms. Application of the error profile extraction to the corrected interferograms shows a practically flat residual error profile, with no more than $0.05 \mathrm{~nm}$ rms deviation.

\section{Parasitic noise spectrum}

As for any photonic instrument, the ultimate noise originates from the photon noise due to the detected photon flux. Additional noise sources may arise from the beamline itself through mirrors vibrations, not to mention vibrations of the whole setup that can be induced by mechanical vibrations transmitted by the ground. In addition, although the translation system (stage+motor) was chosen for an as smooth as possible displacement, it is likely that it adds noise to the spectrum. We have investigated the noise induced by the translation system, i.e., mainly by the scanning motor. To this end, the $633 \mathrm{~nm}$ laser was injected into the VUV interferometer so as to get about $50 \%$ to $75 \%$ modulation, and the resulting spectrum was computed. Three motorization systems were used: (a) a scanning stage (single-ball bearing slides) with integrated motorization system by motor-screwnut (dc current, speed regulation by tachometer, gear speed reductor), (b) a simple scanning stage (double ball bearing slides) with a pushing motor system (dc, no regulation), and (c) same as (b) except for the use of a stepping motor (used at "constant" speed). 


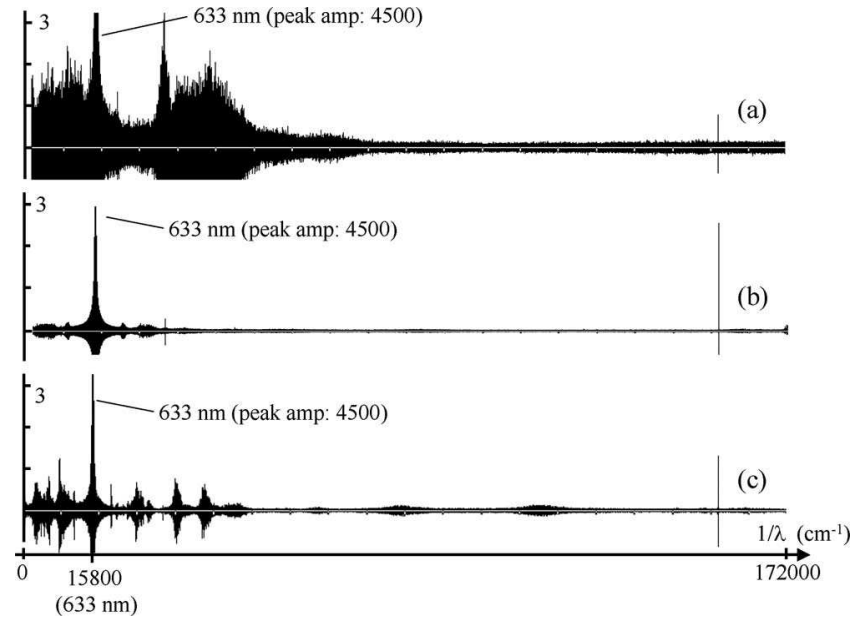

FIG. 5. Checking the noise spectrum added by the scanning system (stage + motor). All spectra show the whole spectral range down to $\sigma$ $=172000 \mathrm{~cm}^{-1}(\lambda=58 \mathrm{~nm}$, right end of the scale; scale is linear in $1 / \lambda)$. The spectrum is computed from an interferogram of the He-Ne laser adjusted to get about 4500 for the peak amplitude. The amplitude range shown is three, as indicated. (a) Stage with integrated motorization (screw-nut, dc motor with speed control); speed: $50 \mu \mathrm{m} / \mathrm{s}$. (b) Simple stage with a separate pushing device (dc motor, no speed control); speed: $40 \mu \mathrm{m} / \mathrm{s}$. (c) As (b), except for a stepping motor; speed: $60 \mu \mathrm{m} / \mathrm{s}$. In all spectra the small peak on the right is a ghost of the laser line, produced by a spurious modulation of the sampling comb with period two samples.

At first glance, as seen in Fig. 5, the spectra show perfect sinc profiles at $\mathrm{He}-\mathrm{Ne}$ spectral position, with maximum amplitude ranging from 4000 to 8000 , depending on the interferometric signal amplitude. However, a closer examination of the low levels (less than 1/1000 of the maximum amplitude) shows side bands spectra as well as different noise levels far from the He-Ne position (Fig. 5). It turns out that obviously, case (b), i.e., a freely driven dc device pushing onto a simple translation stage, produces the best result. Unfortunately, we did not find such a pushing device qualified for UHV operation. As for the future UHV instrument (see Sec. VII), we plan to use the same simple translation stage but driven by a pushing device actuated by a stepping motor with microstep capability and without gear reduction. The results presented hereafter were obtained with solution (a), which was in fact not an issue due to the relatively high photon noise level.

\section{FIRST UV SPECTRUM: PHOTOABSORPTION OF $\mathrm{O}_{2}$ in the $190-200 \mathrm{~nm}$ region}

In order to validate the instrument principle and its implementation in view of being operated as a SR-based absorption facility, we measured our first UV spectrum with a synchrotron beamline as the photon source. The FT prototype was therefore installed in 2003 on the late VUV undulator-based SU5 synchrotron beamline ${ }^{5}$ (Super-ACO, LURE). Due to some noncritical technical problems (related to the vacuum configuration) and a lack of time, we decided to record our first spectrum in air taking advantage of the atmospheric oxygen column over the optical path of SR in between the beamline and the FT spectrometer. More precisely, the horizontal input slit was positioned just after the beamline last $\mathrm{MgF}_{2}$ window (wavelength cutoff around

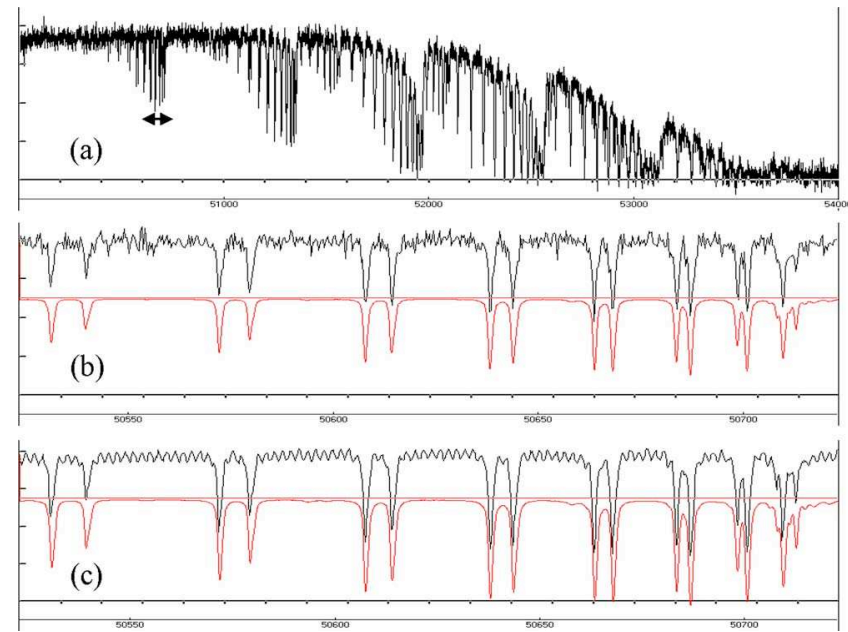

FIG. 6. (Color online) Spectrum of oxygen near $50000 \mathrm{~cm}^{-1}(200 \mathrm{~nm})$ obtained from absorption by atmospheric oxygen. Spectra (a) and (b) were obtained in 2003 on the SU5 beamline at Super-ACO (LURE, Orsay) by averaging 50 spectra, computed from interferograms with $512 \mathrm{~K}$ samples and $26.5 \mathrm{~nm}$ sampling interval (FWHM instrumental width: $0.43 \mathrm{~cm}^{-1}$ ) (see Sec. VI). Spectrum (c) is the first spectrum obtained in 2007 under similar conditions from the DESIRS beamline (SOLEIL) with the upgraded system (see Sec. VII). In this case, 38 interferograms with $1024 \mathrm{~K}$ samples were used with a $24.5 \mathrm{~nm}$ sampling interval $\left(0.23 \mathrm{~cm}^{-1}\right.$ FWHM instrumental width). The sine modulation is due to interferences from the two faces of the last $\mathrm{MgF}_{2}$ window closing the beamline vacuum. (a) shows the spectrum between 50000 and $54000 \mathrm{~cm}^{-1}$. (b) and (c) show a $200 \mathrm{~cm}^{-1}$ bandwidth centered at $50625 \mathrm{~cm}^{-1}$. The bottom trace is a computed spectrum using data from Refs. 34 and 35 (shifted for clarity). Absorption in the computed spectrum was adjusted by trimming the air column length to [(a) and (b)] 150 and (c) $270 \mathrm{~cm}$. Note that experimental spectra are not deconvolved for the instrumental width.

$140 \mathrm{~nm}$ ). The SU5 undulator emits a partially coherent radiation due to interferences of the SR emitted at each wiggle, resulting in a spectral bump with a relative width given by $1 / N$, where $N$ is the number of interfering periods, in the case of the SU5 undulator OPHELIE, ${ }^{32} 1 / N \sim 11 \%$. In addition, as a variable polarization undulator, OPHELIE allows the production of the linear vertical polarization ${ }^{33}$ (polarization $S$ in our case). We did not use the SU5 beamline monochromator dispersion capabilities and worked in the zero order in order to keep as much photon flux as possible (in the order of $5 \times 10^{14}$ photons/s integrated in the whole spectral width). In these conditions, the average detected signal corresponds to a flux of $3.3 \times 10^{10}$ photons/s or $10^{6}$ photons per interferogram sample.

Figure 6 shows the absorption spectrum that we recorded in the Schumann-Runge region of $\mathrm{O}_{2}(B \leftarrow X$ transitions, i.e., ${ }^{3} \Sigma_{u}^{-} \leftarrow{ }^{3} \Sigma_{g}^{-}$). The spectrum is the average of 50 spectra obtained from 50 interferograms (about 3 min recording time per interferogram). From the source slit to the detector, the total path in air at atmospheric pressure is $150 \mathrm{~cm}$, giving a column density of oxygen of about $8.4 \times 10^{20} \mathrm{~cm}^{-2}$. The Schumann-Runge bands of $\mathrm{O}_{2}$ have been extensively studied using a grating-based spectrometer and even more recently by FTS on a synchrotron beamline ${ }^{34}$ for the bands above $\nu^{\prime}=12$. As our spectrum covers the $(1,0)-(7,0)$ bands, we compared our data with published experimental work made by Yoshino et al. ${ }^{35,36}$ Such a comparison can be seen in Fig. 6(b), where we include a computed spectrum based on pub- 
lished data in a restricted band. The two spectra are very similar considering the line positions, shapes, and intensities over the whole considered spectral range. The theoretical instrumental linewidth is $0.43 \mathrm{~cm}^{-1}$ (FWHM), which corresponds to a resolving power of 150000 . The Doppler broadening contribution at room temperature (about $0.11 \mathrm{~cm}^{-1}$ ) can be neglected. Note that the predissociation natural line width of these bands is in general larger than the instrumental linewidth. ${ }^{34}$

Finally, it is noteworthy that photon noise is usually the dominant contribution in the UV and VUV ranges. This was checked both by calculating the expected rms spectral noise from the average detected photon flux for comparison with the actual value and by recording no-signal interferograms to measure the noise added by the detection chain. To summarize, all experimental results show that the FT spectrometer behaves as expected. The resolving power is 150000 at $200 \mathrm{~nm}$, which should translate into 500000 at $60 \mathrm{~nm}$, as it was designed for, allowing the extraction of valuable spectroscopic data in terms of line position, oscillator strength (intensity), and width.

\section{TOWARD IN-VACUUM VUV OPERATION}

The instrument operated in 2003 was built to operate in a vacuum environment for high resolution absorption spectroscopy over the whole VUV range. However, further tests showed that vacuum operation, more precisely clean $U H V$ operation, was an issue for VUV operation regarding the high brightness of a third generation synchrotron source and the very high photon density expected on the reflectors. In such conditions the contamination by hydrocarbons on the reflectors is critical.

This was the main motivation of a general upgrade of the instrument, which was completed at the end of 2007. Although exactly the same design principles are used, we took advantage of this upgrade to enhance the targeted performances of the spectrometer. First, the sampling interval was made adjustable during operation (among a set of ten built in values) by allowing the fan parameter $p$ to be changed in vacuum while operating the spectrometer. It is therefore possible to adapt the Nyquist frequency (and therefore the resolution) to the spectrum being recorded, as mentioned in Sec. III B. Second, the scan range was increased to allow at least $1024 \mathrm{~K}$ samples per interferogram whatever the sampling interval is. Finally, the spectral range was extended down to $\lambda=40 \mathrm{~nm}(\sim 30 \mathrm{eV})$ by using $\mathrm{SiC}$ instead of Pt for the VUV reflecting coating.

The upgraded instrument is currently installed and tested on the new French synchrotron source SOLEIL, more precisely on the VUV undulator-based DESIRS beamline, ${ }^{37}$ of which it will become a permanent endstation. As an example of its actual performances we present in Fig. 6(c) the same $\mathrm{O}_{2}$ spectrum as in Sec. VI but recorded with $1 \times 10^{6}$ samples, leading to a theoretical instrumental linewidth of $0.23 \mathrm{~cm}^{-1}$ (FWHM) [Fig. 6(c)]. This figure should translate in the VUV range to an intrinsic resolving power close to $1 \times 10^{6}$. In addition, the average detected signal is improved by a factor 20 as it can be seen on the much higher signal to noise ratio as compared to Fig. 6(b). Especially in Fig. 6(c), one can clearly observe the small modulation due to interferences between the faces of the last $\mathrm{MgF}_{2}$ window. The same modulations should be observed in Fig. 6(b) but are smeared out in the noise. The commissioning of the upgraded FTS in the VUV range is in progress and will be the object of a forthcoming paper.

\section{CONCLUSION}

We designed and built a FT spectrometer that opens the way toward very high resolution absorption spectroscopy in the VUV range well below the beam splitter limit (140 nm) with potentially unprecedented resolving powers, as compared to grating-based spectrometer, over a very wide spectral range. An important part of this project was the design and manufacture of the control and sampling system. A special care has been given to the postacquisition interferogram treatment in order to detect and correct for possible residual sampling errors. After having recorded the apparatus function in the visible with a $\mathrm{He}-\mathrm{Ne}$ laser showing a near perfect behavior, we have been able to record $\mathrm{O}_{2}$ absorption spectrum in the near VUV region, showing that very accurate spectroscopic data could be obtained from such an instrument. Both experimental results were very encouraging for the next step of our project. The instrument was rebuilt to be qualified to an UHV environment with an improved resolving power and an extended operating range down to $\lambda$ $=40 \mathrm{~nm}$. The preliminary results obtained on the DESIRS VUV beamline show a nominal behavior regarding the accuracy of data acquisition and a marked increase in the signal to noise ratio partly due to the high brilliance of the undulator beamline coupled with the third generation synchrotron SOLEIL. The coupling of the upgraded FTS to the undulator-based beamline DESIRS leads to a unique facility dedicated to ultrahigh resolution absorption spectroscopy on a broad VUV spectral range open to the general users community.

\section{ACKNOWLEDGMENTS}

The authors acknowledge the invaluable skill and savoir faire of the "optical surface and component" group from the Laboratoire Charles Fabry for the optical parts fabrication and metrology. We are also indebted to the general technical staff of the LURE and SOLEIL facilities.

This work has been supported by the ANR (Agence Nationale de la Recherche) under Grant No. 05-BLAN-0364.

\footnotetext{
${ }^{1}$ Note that in the following, "VUV range" refers to the 5-40 eV range (250-30 nm), a range usually covered by normal incidence monochromators and which corresponds to valence and inner-valence-shell excitations of molecules.

${ }^{2}$ J. Chamberlain, The Principles of Interferometric Spectroscopy (Wiley, New York, 1979).

${ }^{3}$ A. Thorne, J. Anal. At. Spectrom. 13, 407 (1998).

${ }^{4}$ See, for instance, U. Hollenstein, H. Palm, and F. Merkt, Rev. Sci. Instrum. 71, 4023 (2000) and references therein.

${ }^{5}$ L. Nahon, C. Alcaraz, J. L. Marlats, B. Lagarde, F. Polack, R. Thissen, D. Lepere, and K. Ito, Rev. Sci. Instrum. 72, 1320 (2001).

${ }^{6}$ A. Thorne, C. J. Harris, I. Winne-Jones, R. C. M. Learner, and C. Cox, J. Phys. E 20, 54 (1987).

${ }^{7}$ K. Ito, K. Maeda, Y. Murioka, and T. Namioka, Appl. Opt. 28, 1813
} 
(1989).

${ }^{8}$ J. C. Pickering, Vib. Spectrosc. 29, 27 (2002).

${ }^{9}$ G. Nave, S. Johansson, and A. Thorne, J. Opt. Soc. Am. B 14, 1035 (1997).

${ }^{10}$ A. Thorne, Phys. Scr. t65, 31 (1996).

${ }^{11}$ T. Haga, M. C. K. Tinone, M. Shimada, T. Ohkubo, and A. Ozawa, J. Synchrotron Radiat. 5, 690 (1998).

${ }^{12}$ L. B. Da Silva, T. W. Barbee, Jr., R. Cauble, P. Celliers, D. Ciarlo, J. C. Moreno, S. Mrowka, J. E. Trebes, A. S. Wan, and F. Weber, Appl. Opt. 34, 6389 (1995).

${ }^{13}$ P. P. Naulleau, C. H. Cho, E. M. Gullikson, and J. Bokor, J. Synchrotron Radiat. 7, 405 (2000).

${ }^{14}$ J. Filevich, K. Kanizay, M. C. Marconi, J. L. A. Chilla, and J. J. Rocca, Opt. Lett. 25, 356 (2000).

${ }^{15}$ M. R. Howells, K. Frank, Z. Hussain, E. J. Moler, T. Reich, D. Moller, and D. A. Shirley, Nucl. Instrum. Methods Phys. Res. A 347, 182 (1994).

${ }^{16}$ H. Yin, M. Wang, M. Strom, and J. Nordgren, Nucl. Instrum. Methods Phys. Res. A 451, 529 (2000).

${ }^{17}$ M. Kovacev, S. V. Fomichev, E. Priori, Y. Mairesse, H. Merdji, P. Monchicourt, P. Breger, J. Norin, A. Persson, A. L'Huillier, C.-G. Wahlström, B. Carré, and P. Salieres, Phys. Rev. Lett. 95, 223903 (2005).

${ }^{18}$ J. Svatos, D. Joyeux, D. Phalippou, and F. Polack, Opt. Lett. 18, 1367 (1993).

${ }^{19}$ J. J. Rocca, C. H. Moreno, M. C. Marconi, and K. Kanizay, Opt. Lett. 24, 420 (1999).

${ }^{20}$ F. Albert, P. Zeitoun, P. Jaegle, D. Joyeux, M. Boussoukaya, A. Carillon, S. Hubert, G. Jamelot, A. Klisnick, D. Phalippou, D. Ros, and A. ZeitounFakiris, Phys. Rev. B 60, 11089 (1999).

${ }^{21}$ D. Joyeux, F. Polack, and D. Phalippou, Rev. Sci. Instrum. 70, 2921 (1999).

${ }^{22}$ H. Tang, O. Guilbaud, G. Jamelot, D. Ros, A. Klisnick, D. Joyeux, D. Phalippou, M. Kado, N. Nishikino, M. Nishikino, K. Sukegawa, M. Ishino, K. Nagashima, and H. Daido, Appl. Phys. B: Lasers Opt. 78, 975
(2004).

${ }^{23}$ F. Polack, D. Joyeux, J. Svatos, and D. Phalippou, Rev. Sci. Instrum. 66, 2180 (1995).

${ }^{24}$ M. Born and E. Wolf, Principle of Optics, 5th ed. (Pergamon, New York, 1975).

${ }^{25}$ C. Chang, P. Naulleau, and D. Attwood, Appl. Opt. 42, 2506 (2003).

${ }^{26}$ S. Chandra and R. S. Rohde, Appl. Opt. 21, 1533 (1982).

${ }^{27} \mathrm{~N}$. De Oliveira, "Etude et realisation d'un spectromètre par transformation de Fourier, sans lame separatrice, pour le domaine spectral VUV-EUV," Ph.D. thesis, Universite Pierre et Marie Curie, 2001.

${ }^{28}$ D. L. Windt, W. C. Cash, Jr., M. Scott, P. Arendt, B. Newnam, R. F, Fisher, and A. B. Swartzlander, Appl. Opt. 27, 246 (1988).

${ }^{29}$ M. L. Forman, W. H. Steel, and G. A. Vanasse, J. Opt. Soc. Am. 56, 59 (1966).

${ }^{30}$ N. De Oliveira, D. Joyeux, D. Phalippou, and F. Polack, Surf. Rev. Lett. 9, 655 (2002).

${ }^{31}$ N. De Oliveira, D. Joyeux, D. Phalippou, J. C. Rodier, L. Nahon, F. Polack, and M. Vervloët, AIP Conf. Proc. 879, 447 (2007).

${ }^{32}$ L. Nahon and C. Alcaraz, Appl. Opt. 43, 1024 (2004).

${ }^{33}$ L. Nahon, R. Thissen, C. Alcaraz, M. Corlier, P. Peaupardin, F. Marteau, O. Marcouillé, and P. Brunelle, Nucl. Instrum. Methods Phys. Res. A 447, 569 (2000).

${ }^{34}$ T. Matsui, A. S.-C. Cheung, K. W.-S. Leung, K. Yoshino, W. H. Parkinson, A. P. Thorne, J. E. Murray, K. Ito, and T. Imajo, J. Mol. Spectrosc. 219, 45 (2003).

${ }^{35}$ K. Yoshino, J. R. Esmond, A. S.-C. Cheung, D. E. Freeman, and W. H. Parkinson, Planet. Space Sci. 40, 185 (1992) (http://www.cfa.harvard.edu/ amp/tools.html).

${ }^{36}$ K. Yoshino, D. E. Freeman, J. R. Esmond, and W. H. Parkinson, Planet. Space Sci. 31, 339 (1983).

${ }^{37}$ DESIRS beamline website: www.synchrotron-soleil.fr/portal/page/portal/ Recherche/LignesLumiere/DESIRS. 